\title{
Is Backscatter Link Stronger than Direct Link in Reconfigurable Intelligent Surface-Assisted System?
}

\author{
Wenjing Zhao, Gongpu Wang, Saman Atapattu, Senior Member, IEEE, \\ Theodoros A. Tsiftsis, Senior Member, IEEE, and Chintha Tellambura, Fellow, IEEE,
}

\begin{abstract}
This letter considers integrating a backscatter link with a reconfigurable intelligent surface to enhance backscatter communication while assisting the direct communication. We derive the probability that the backscatter channel dominates in the composite channel. This probability is a useful performance measure to determine the number of reflectors. Since the exact probability lacks a closed-form solution, we develop two approximations by modeling the gain of the backscatter link with a Gaussian or Gamma distribution. We found that these approximations match well with the exact value. Importantly, with a well-designed number of reflectors, the channel gain of the backscatter link may be always stronger than that of the direct one.
\end{abstract}

Index Terms-Backscatter communication, reconfigurable intelligent surface (RIS), parameter design.

\section{INTRODUCTION}

Reconfigurable intelligent surface (RIS) technology [1] enables wireless charging, remote sensing and unprecedented data transmission in line with the vision of future wireless communications that are low-cost, energy-efficient and connectivity-ubiquitous. RIS consists of many nearly passive reflectors, each of which can sensibly interact with the impinging signal in a software-controlled way. Specifically, each reflector can vary the phase of the incident signal with the perceived channel state information (CSI) without requiring special energy source, in order to realize a high-performance communication link. Moreover, RIS can be easily embedded into objects such as clothing, furniture, walls, and building facades.

This study is supported in part by Key Laboratory of Universal Wireless Communications (BUPT), Ministry of Education, P.R.China under Grant KFKT-2018104, in part by the Natural Science Foundation of China (NSFC) under Grants 61571037, 61871026 and U1834210, in part by NFSC Outstanding Youth under Grant 61725101, and in part by the Australian Research Council (ARC) through the Discovery Early Career Researcher (DECRA) Award under Grant DE160100020. (Corresponding author: Gongpu Wang)

W. Zhao and G. Wang are with Beijing Key Lab of Transportation Data Analysis and Mining, School of Computer and Information Technology, Beijing Jiaotong University, China (e-mail: \{wenjingzhao, gpwang\}@bjtu.edu.cn).

S. Atapattu is with the Department of Electrical and Electronic Engineering, The University of Melbourne, Parkville, VIC 3010, Australia (e-mail: saman.atapattu@unimelb.edu.au).

T. A. Tsiftsis is with the School of Intelligent Systems Science and Engineering, Jinan University, Zhuhai 519070, China (email: theo_tsiftsis@jnu.edu.cn).

C. Tellambura is with the Department of Electrical and Computer Engineering, University of Alberta, Edmonton, AB T6G 1H9, Canada (e-mail: chintha@ece.ualberta.ca).
State-of-the-art research on RIS includes data transmission [1]-[3], energy efficiency of multi-user communications [4], beamforming design [5]-[7], user assignment in distributed RIS systems [8], secure [9] and ultra-reliable communication [10]. Existing RIS techniques can provide extra paths to aid transmission from the transmitter to the receiver. However, it is often assumed that the RIS device itself cannot transmit extra information to the receiver [4]-[7], [9], [11]. In contrast, references [2], [3] show that a RIS device can realize information transfer by adopting spatial index modulation. By virtue of the information transmission feature of the RIS, we integrate backscatter technology with RIS. We thus introduce a new paradigm that can not only enable RIS to aid an ongoing data transmission, but also transmit its own binary signals or sensor signals to the receiver by performing binary modulation through RIS, embedding sensors into RIS, or connecting RIS to surrounding sensors through wires [10], [12]. Specifically, RIS synchronizes with the transmitter and conveys data $\{0$, $1\}$ to the receiver. In this way, loading information ' 1 ' or ' 0 ' means that RIS is in a reflective or absorptive state. The key performance measure that helps of the design of this overall system is the probability that the gain of backscatter link is stronger than that of direct one. We derive this measure in both exact and approximate forms and provide numerical and simulations results. By using this measure, one can choose the appropriate number of reflectors to ensure high-quality communications even under extremely poor direct channel conditions.

Notation: For random variable $(\mathrm{RV}) X, f_{X}(\cdot)$ and $F_{X}(\cdot)$ denote the probability density function (PDF) and cumulative distribution function, respectively. A complex or real Gaussian RV with mean $\mu$ and variance $\sigma^{2}$ is denoted by $\mathcal{C N}\left(\mu, \sigma^{2}\right)$ or $\mathcal{N}\left(\mu, \sigma^{2}\right)$. The $\operatorname{Exp}(\lambda)$ density is $f_{X}(x)=\frac{1}{\lambda} e^{-\frac{x}{\lambda}}, x \geq 0$. The $\operatorname{Ral}(1)$ density is $f_{X}(x)=2 x e^{-x^{2}}, x \geq 0$. We write $X \sim \operatorname{Gamma}(\alpha, \beta)$ if $f_{X}(x)=\frac{\beta^{\alpha}}{\Gamma(\alpha)} x^{\alpha-1} e^{-\beta x}, \quad x \geq 0$.

\section{System Model}

Fig. 1 shows the proposed system consists of a backscatter link, direct link, one RF source, one receiver and one RIS. The RIS unit comprises of $K$ passive reflectors $\{1, \cdots, K\}$, and also includes a controller for CSI acquisition and information transmission [13]. When the RF source transmits its data signal $s(n)$, whose power is $\sigma_{s}^{2}$, to the receiver, the RIS will also receive the same signal and then adjust the phase 




Fig. 1. Integrated RIS and backscatter technology.

$\theta_{k} \in[0,2 \pi)$ of reflector $k \in\{1, \cdots, K\}$ based upon CSI. Moreover, by performing binary modulation or connecting RIS with surrounding sensors through wires, RIS can send its data $x(n) \in\{0,1\}$ to the receiver by reflecting or absorbing the incident signal $s(n)$.

Let $h_{s r}, h_{s t, k}$ and $h_{t r, k}$ denote the channel coefficients from $\mathrm{RF}$ source to receiver, from RF source to reflector $k$, and from reflector $k$ to receiver, respectively. ${ }^{1}$ Denote the distances from $\mathrm{RF}$ source to receiver, from RF source to reflector $k$, and from reflector $k$ to receiver by $d_{s r}, d_{s t}$ and $d_{t r}$, respectively. All these small-scale fading channel coefficients are modeled as independent and identically distributed $\mathcal{C N}(0,1)$ variables [15]. In other words, this model is equivalent to small-scale Rayleigh fading. We also denote by $\alpha$ the large-scale path loss factor for all the links. The signal received at reflector $k=1, \ldots, K$ can be expressed as

$$
y_{k}(n)=\frac{h_{s t, k}}{\sqrt{d_{s t}^{\alpha}}} s(n) .
$$

With the phase adjustment based on CSI for specific purposes, the signal backscattered by the $k$ th reflector is

$$
y_{b, k}(n)=\eta y_{k}(n) x(n) e^{j \theta_{k}},
$$

where $\eta$ is the RIS attenuation factor. The signal arrived at the receiver is the superposition of the RF source signal and all the reflected signals and can be expressed as

$$
y(n)=\frac{h_{s r}}{\sqrt{d_{s r}^{\alpha}}} s(n)+\sum_{k=1}^{K} y_{b, k}(n)+w(n),
$$

where $w(n)$ is additive white Gaussian noise with variance $\sigma_{w}^{2}$. By substituting (1) and (2) into (3), the received signal (3) can be rewritten as

$y(n)=\left(\frac{h_{s r}}{\sqrt{d_{s r}^{\alpha}}}+\eta \sum_{k=1}^{K} \frac{h_{s t, k} h_{t r, k}}{\sqrt{d_{s t}^{\alpha} d_{t r}^{\alpha}}} e^{j \theta_{k}} x(n)\right) s(n)+w(n)$.

\footnotetext{
${ }^{1}$ Channels can be estimated in advance via sub-arrays techniques [13] or deep learning techniques [14].
}

\section{RIS PARAMETER DESIGN}

With RIS assistance, when $x(n)=1$ is transmitted, the maximized instantaneous signal-to-noise (SNR) at the receiver is $\left(d_{s r}^{-\frac{\alpha}{2}}\left|h_{s r}\right|+\eta d_{s t}^{-\frac{\alpha}{2}} d_{t r}^{-\frac{\alpha}{2}} \sum_{k=1}^{K}\left|h_{s t, k} h_{t r, k}\right|\right)^{2} \frac{\sigma_{s}^{2}}{\sigma_{w}^{2}}$ when $\theta_{k}=\left(\angle h_{s r}-\angle h_{s t, k} h_{t r, k}\right) \bmod 2 \pi$ holds. Here, $\angle x$ and $x$ mod $y$ are the phase of $x$ and the remainder after the division of $x$ by $y$, respectively.

Backscatter link, also called multiplicative multiple access channel [16], carries information from both RF source and RIS whereas direct link merely loads RF source information. Moreover, RIS can be used to enhance the direct link communication while transmitting its own information to the receiver [2]. Therefore, it is wise to strengthen the transmission of backscatter link without affecting that of direct link or even make backscatter link main path in the composite link $d_{s r}^{-\frac{\alpha}{2}}\left|h_{s r}\right|+\eta d_{s t}^{-\frac{\alpha}{2}} d_{t r}^{-\frac{\alpha}{2}} \sum_{k=1}^{K}\left|h_{s t, k} h_{t r, k}\right|$. Due to the effect of channel fading, it is not true that the signal quality from backscatter link (i.e., from RF source to RIS to receiver) is always stronger than that from direct link (i.e., from RF source to receiver). We measure this characteristic by assuming that the probability of the channel gain of the backscatter link is greater than that of the direct link. Mathematically, it can be expressed as

$$
P=\operatorname{Pr}\left(\left|\frac{h_{s r}}{\sqrt{d_{s r}^{\alpha}}}\right|<\left|\eta \frac{\sum_{k=1}^{K} h_{s t, k} h_{t r, k} e^{j \theta_{k}}}{\sqrt{d_{s t}^{\alpha} d_{t r}^{\alpha}}}\right|\right) .
$$

How can such a case be improved? Next, we will analyze how to design the number of reflectors $K$ to deal with this.

\section{A. Intelligent Transmission}

Due to $\left|\sum_{k=1}^{K} h_{s t, k} h_{t r, k} e^{j \theta_{k}}\right|=\sum_{k=1}^{K}\left|h_{s t, k}\right|\left|h_{t r, k}\right|$ in the case of perfect CSI, (5) can be simplified as

$$
P=\operatorname{Pr}\left(d_{s r}^{-\frac{\alpha}{2}}\left|h_{s r}\right|<\eta d_{s t}^{-\frac{\alpha}{2}} d_{t r}^{-\frac{\alpha}{2}} \sum_{k=1}^{K}\left|h_{s t, k} h_{t r, k}\right|\right) .
$$

Let us define variable $G_{d} \triangleq\left|h_{s r}\right|$ and variable $G_{b} \triangleq$ $\sum_{k=1}^{K}\left|h_{s t, k}\right|\left|h_{t r, k}\right|$. Then, (6) is equivalent to

$$
P=\operatorname{Pr}\left(G_{d}<c_{d} G_{b}\right)
$$

where

$$
c_{d} \triangleq \eta d_{s r}^{\frac{\alpha}{2}} d_{s t}^{-\frac{\alpha}{2}} d_{t r}^{-\frac{\alpha}{2}} .
$$

Then, we can rewrite (7) as

$$
\begin{aligned}
P & =\int_{0}^{\infty} f_{G_{b}}(y) \int_{0}^{c_{d} y} f_{G_{d}}(x) d x d y, \\
& =\int_{0}^{\infty} f_{G_{d}}(x)[1-\underbrace{\int_{0}^{\frac{x}{c_{d}}} f_{G_{b}}(y) d y}_{F_{G_{b}}\left(x / c_{d}\right)}] d x,
\end{aligned}
$$

where (9) and (10) hold because $G_{d}$ and $G_{b}$ are independent.

Clearly, we have $G_{d} \sim \operatorname{Ral}(1)$. From (9) and (10), the key point is to determine $F_{G_{b}}(x)$ or $f_{G_{b}}(x)$. Because the PDF $f_{G_{b}}(x)$ is analytically intractable, we derive the exact probability using (10). 
1) Exact computation: We can readily show that the characteristic function of $G_{b}$ is given by

$$
\begin{aligned}
\varphi(t) & =\left[\int_{0}^{\infty} 4 x K_{0}(2 x) e^{j t x} d x\right]^{K} \\
& =\left[\frac{j 2 \pi t+4 \sqrt{4+t^{2}}-4 t \operatorname{arcsinh}\left(\frac{t}{2}\right)}{\left(4+t^{2}\right)^{\frac{3}{2}}}\right]^{K},
\end{aligned}
$$

where (11) follows by [17, eq. (6.45)], and (12) follows by [18, eq. (6.624)] and [18, eq. (1.622)]. Here, $j=\sqrt{-1}$, $K_{\alpha}(x)$ is the modified Bessel function of the second kind [18, eq. (8.432)], and $\operatorname{arcsinh}(x)$ is the inverse hyperbolic sine function. From the Gil-Pelaez lemma [19] and for certain value $G_{d}=x$, we can thus compute

$$
F_{G_{b}}\left(\frac{x}{c_{d}}\right)=\frac{1}{2}-\frac{1}{\pi} \int_{0}^{\infty} \Im\left[\frac{e^{-j t x / c_{d}} \varphi(t)}{t}\right] d t,
$$

where $\Im[x]$ denotes the imaginary part of $x$. Based on (10) and then averaging (13) over the PDF of $G_{d}$, we find that

$$
\begin{aligned}
P & =1-\int_{0}^{\infty} F_{G_{b}}\left(x / c_{d}\right) f_{G_{d}}(x) d x \\
& =\frac{1}{2}+\frac{1}{\pi} \int_{0}^{\infty} \Im\left[\frac{\left(1-\sqrt{\pi} t_{1} e^{\left.-t_{1}^{2}\left[\operatorname{erfi}\left(t_{1}\right)+j\right]\right) \varphi(t)}\right.}{t}\right] d t,
\end{aligned}
$$

where $t_{1}=\frac{t}{2 c_{d}}$ and the imaginary error function $\operatorname{erfi}(x)$ is defined by erfi $(x)=\frac{2}{\sqrt{\pi}} \int_{0}^{x} e^{t^{2}} d t$.

Since exact expression (14) has no closed-form solution, we will develop two approximations for $P$. The problem is that a closed-form expression for $f_{G_{b}}(y)$ appears intractable. Hence, we will utilize Gaussian and Gamma approximations.

2) Gaussian approximation: The central limit theorem suggests that $G_{b}$ is Gaussian for large $K$, namely, $\mathcal{N}\left(\mu_{b}, \sigma_{b}^{2}\right)$ where the mean and the variance can be separately derived as

$$
\left\{\begin{array}{l}
\mu_{b} \triangleq \mathbb{E}\left\{G_{b}\right\}=\frac{\pi}{4} K, \\
\sigma_{b}^{2} \triangleq \mathbb{E}\left\{\left|G_{b}-\mu_{b}\right|^{2}\right\}=\left(1-\frac{\pi^{2}}{16}\right) K .
\end{array}\right.
$$

Thus, by using this Gaussian distribution on (9) and evaluating the resulting integral, we find that

$$
\begin{aligned}
P= & -\frac{1}{2 \sqrt{2 c_{d}^{2} \sigma_{b}^{2}+1}} \operatorname{erfc}\left(-\frac{\mu_{b}}{\sqrt{4 c_{d}^{2} \sigma_{b}^{4}+2 \sigma_{b}^{2}}}\right) \\
& \times e^{-\frac{\mu_{b}^{2}}{2 \sigma_{b}^{2}}+\frac{\mu_{b}^{2}}{4 c_{d}^{2} \sigma_{b}^{4}+2 \sigma_{b}^{2}}}+\frac{1}{2} \operatorname{erfc}\left(-\frac{\mu_{b}}{2 \sqrt{\sigma_{b}^{2}}}\right) .
\end{aligned}
$$

This derivation is based upon [18, eq. (3.323.2)], and $\operatorname{erfc}(x)$ is the complementary error function [18, eq. (8.250.4)].

3) Gamma approximation: For small $K$, the Gaussian approximation of $G_{b}$ may not be accurate. To tackle this problem, we consider $G_{b}$ to be distributed as $\operatorname{Gamma}\left(\beta_{1}, \beta_{2}\right)$. In (15), we have the exact mean and variance of $G_{b}$. We match those two and get $\mu_{b}=\frac{\beta_{1}}{\beta_{2}}$ and $\sigma_{b}^{2}=\frac{\beta_{1}}{\beta_{2}^{2}}$. Thus, we find

$$
\beta_{1}=\frac{K \pi^{2}}{16-\pi^{2}} \quad \text { and } \quad \beta_{2}=\frac{4 \pi}{16-\pi^{2}} .
$$

Now, with [18, eq. (3.462.1)], (9) can be evaluated as

$$
P=1-\frac{\beta_{2}^{\beta_{1}}}{2^{\frac{\beta_{1}}{2}} c_{d}^{\beta_{1}}} e^{\frac{\beta_{2}^{2}}{8 c_{d}^{2}}} D_{-\beta_{1}}\left(\frac{\beta_{2}}{\sqrt{2 c_{d}^{2}}}\right),
$$

where $D_{p}(z)$ is the parabolic cylinder function [18, eq. (9.240)].

Remark 1: The simulation results in Section IV suggest that the Gamma-based approximation, namely (18), is more accurate. However, the Gaussian-based approximation, (16), is rather easy to compute. Moreover, the computation of the parabolic cylinder function for large $K$ may encounter difficulties in practice. For this reason, we next develop another approximation suitable for large $K$ scenarios.

Remark 2: With the aid of [18, eq. (9.229.2)] and [18, eq. (9.240)], for large $K$, (18) can be approximated by

$$
P=1-\left(\frac{\beta_{2}}{2 c_{d}} e^{\frac{1}{2}-\frac{1}{2} \log t_{2}}\right)^{\beta_{1}} e^{\frac{1}{4} \log t_{2}-\frac{\beta_{2}}{c_{d}} \sqrt{t_{2}}+\frac{\beta_{2}^{2}}{8 c_{d}^{2}},}
$$

where $t_{2}=\frac{\beta_{1}}{2}-\frac{1}{4}$. This approximation not only eliminates the need to compute the parabolic cylinder function, but also allows for $P$ to be computed effectively for large $K$.

Remark 3: When channel coefficients $h_{s t, k}$ and $h_{t r, k}$ are distributed according to the Rician fading model, we can still model the probability density of the RV $G_{b}$ with the above Gaussian and Gamma approximations, just with different Gaussian and Gamma parameters, i.e., $\mu_{b}, \sigma_{b}^{2}, \beta_{1}$ and $\beta_{2}$. The details are omitted.

\section{B. Blind Transmission}

When RIS has no knowledge of CSI, it merely changes phases randomly. In this case, it may be unable to work effectively. To reduce energy consumption at RIS, we assume that there is no phase change at RIS, namely $\theta_{k}=0, \forall k$. Probability (5) can then be measured equivalently by

$$
P=\operatorname{Pr}\left(\left|\frac{h_{s r}}{\sqrt{d_{s r}^{\alpha}}}\right|^{2}<\left|\eta \frac{\sum_{k=1}^{K} h_{s t, k} h_{t r, k}}{\sqrt{d_{s t}^{\alpha} d_{t r}^{\alpha}}}\right|^{2}\right) .
$$

With large $K$, via the central limit theorem, we find that $\sum_{k=1}^{K} h_{s t, k} h_{t r, k} \sim \mathcal{C N}(0, K)$. Define $P_{d} \triangleq\left|h_{s r}\right|^{2}$ and $P_{b} \triangleq$ $\left|\sum_{k=1}^{K} h_{s t, k} h_{t r, k}\right|^{2}$. Then, $P_{d} \sim \operatorname{Exp}(1)$ and $P_{b} \sim \operatorname{Exp}(K)$. Similar to (9), probability (19) can be derived as

$$
\begin{aligned}
P & =\operatorname{Pr}\left(P_{d}<c_{d}^{2} P_{b}\right)=\int_{0}^{\infty} \frac{1}{K} e^{-\frac{y}{K}} \int_{0}^{c_{d}^{2} y} e^{-x} d x d y \\
& =\frac{K c_{d}^{2}}{K c_{d}^{2}+1} .
\end{aligned}
$$

Suppose that $P>\vartheta$ is required where $\vartheta$ is a suitable threshold probability. According to (20), to set $P>\vartheta$ is equivalent to

$$
K>\frac{\vartheta}{c_{d}^{2}(1-\vartheta)}
$$

From (20) or (21), if the gain of the backscatter link is completely stronger than that of the direct link, i.e., $\vartheta=1, K$ goes to $\infty$. 



Fig. 2. PDF $f_{G_{b}}(x)$.

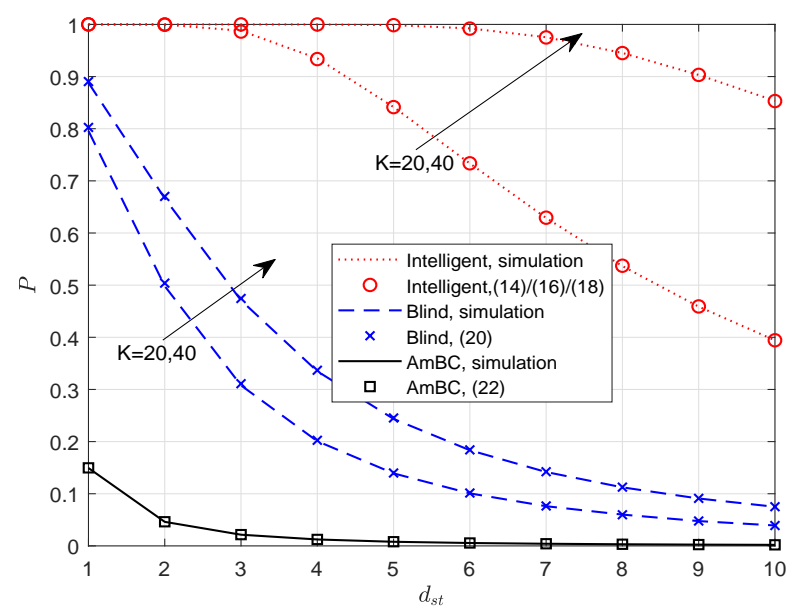

Fig. 3. Probability $P$ versus distance $d_{s t}$.

Remark 4: In an ambient backscatter communication (AmBC) system [20] (i.e., $K=1$ and $\theta_{1}=0$ ), we have

$$
P=1-\frac{1}{c_{d}} e^{\frac{1}{2 c_{d}^{2}}} W_{-\frac{1}{2}, 0}\left(\frac{1}{c_{d}^{2}}\right),
$$

where $W_{\lambda, \mu}(\cdot)$ is the Whittaker function [18, eq. (9.223)].

\section{Numerical And Simulation Results}

We provide simulation results to verify the results derived in Section III. We set $\eta=0.4, d_{t r}=7.1 \mathrm{~m}$ and $d_{s r}=8 \mathrm{~m}$.

Fig. 2 shows the PDF $f_{G_{b}}\left(G_{b}\right)$. We see that Gamma distribution with parameters in (15) can match the simulated exact distribution well for both small and large $K$. Although the Gaussian approximation with the parameters given in (17) does not fit as well as the Gamma one for small $K$, it is quite accurate for large $K$.

Fig. 3 depicts the probability $P$ versus distance $d_{s t}$ when $\alpha=2, K=20$ and $K=40$. Since we found that the curves of (14), (16) and (18) coincide with the simulations, only the curves of (14) are kept. All simulations are statistically averaged over $10^{6}$ independent runs. For comparative purposes,
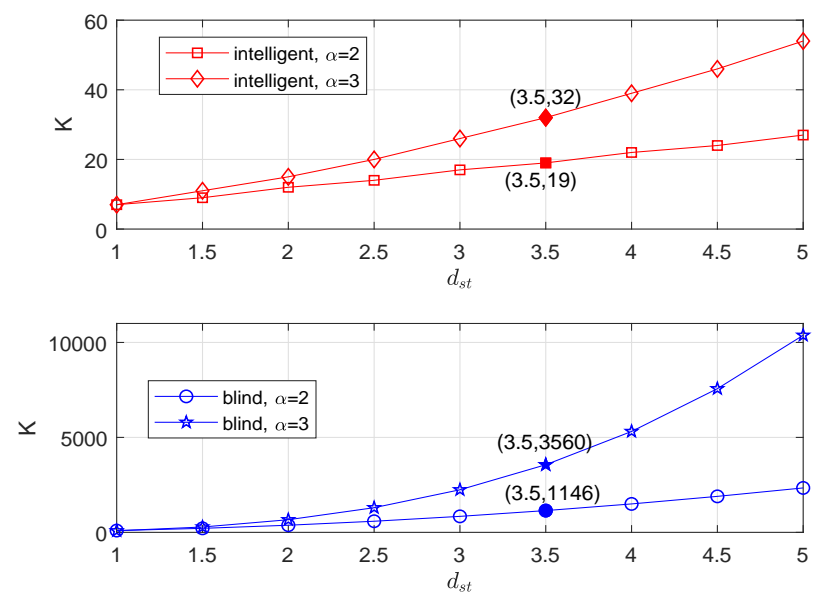

Fig. 4. Minimum number of reflectors required $K$ versus distance $d_{s t}$ when $P \geq 0.95$.
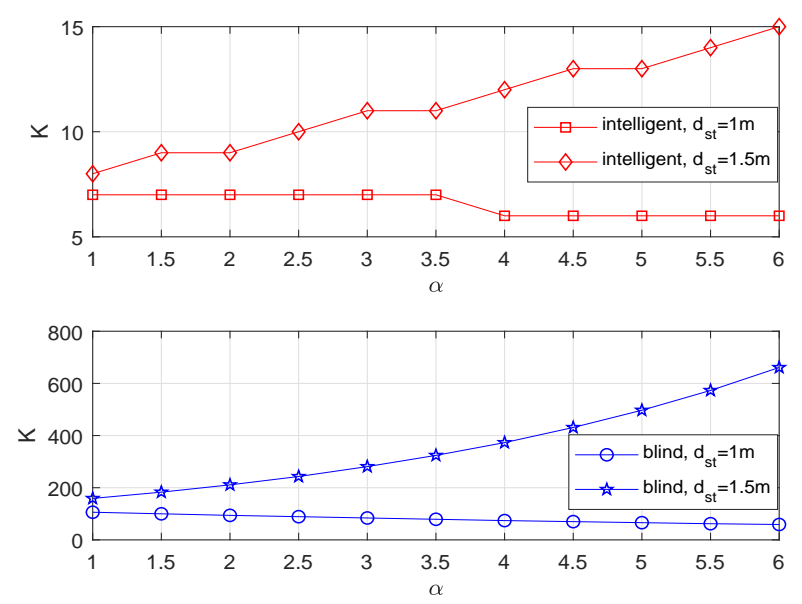

Fig. 5. Minimum number of reflectors required $K$ versus path loss exponent $\alpha$ when $P \geq 0.95$.

the probability (22) in AmBC system is also simulated. In Fig. 3, the simulation results totally match the corresponding theoretical expressions in all cases, which validates the derivations (14), (16), (18), (20) and (22). All probabilities decrease with increasing distance $d_{s t}$ or decreasing $K$. These results show that the optimal placement of RIS should be as close as possible to the RF source. Moreover, the probability in the intelligent scenario is greater than that in the blind scenario and can reach 1 at a large $K=40$. Thus, just well-designed number $K$ may make the backscatter channel the main path.

Figs 4 and 5 separately shed some light on the minimum number $K$ of reflectors required in the intelligent transmission scene versus distance $d_{s t}$ and loss exponent $\alpha$ when $P \geq 0.95$. In Fig. 4, the path loss exponent can be $\alpha=2$ or $\alpha=3$, and the distance can be $d_{s t}=1 \mathrm{~m}$ and $d_{s t}=1.5 \mathrm{~m}$ in Fig. 5. As expected, more reflectors are needed at RIS when increasing distance $d_{s t}$. For a larger path loss factor $\alpha$, the number of reflectors witnesses an upward trend in the case of $\frac{d_{s r}}{d_{s t} d_{t r}}<1$ (e.g., $d_{s t}=1.5 \mathrm{~m}$ ) while there is a completely 
opposite trend for $\frac{d_{s r}}{d_{s t} d_{t r}}>1$ (e.g., $d_{s t}=1 \mathrm{~m}$ ), which can be confirmed from (20). In the intelligent transmission situation, the minimum number of reflectors required is $K=19$ and $K=32$ when $d_{s t}=3.5 \mathrm{~m}$ in the case of $\alpha=2$ and $\alpha=3$, respectively. Under the same parameter configuration and according to (21), the minimum number of reflectors required for blind transmission is $K=1146$ and $K=3560$, respectively, which is separately over 60 and 111 times that of intelligent transmission.

\section{CONClusion AND Future WORK}

This study is the first to integrate the RIS paradigm with backscatter communications. RIS helps to enhance the quality of both the backscatter communication and direct-link communication. The system is characterized by the probability that the channel gain of the backscatter link is greater than that of the direct link. Since the exact probability expression lacks a closed-form solution, we have developed several approximations. From our analysis and numerical results, the key finding is that a reasonably designed number of reflectors can make the backscatter communication link dominate the composite channel. Thus, even without a line of sight link, RIS can achieve a high-capacity wireless transmission. In addition, several future works are immediately evident. First, channel coding and beamforming schemes for this system can be studied. Second, the parameter design of RIS with limited resolution, which in fact results in the loss of channel SNR, should be analyzed. Third, the effect of imperfect CSI should be investigated.

\section{REFERENCES}

[1] S. Hu, F. Rusek, and O. Edfors, "Beyond massive MIMO: The potential of data transmission with large intelligent surfaces," IEEE Trans. Signal Processing, vol. 66, no. 10, pp. 2746-2758, May 2018.

[2] W. Yan, X. Yuan, and X. Kuai, "Passive beamforming and information transfer via large intelligent surface," IEEE Wireless Commun. Letters, pp. 1-1, 2019.

[3] E. Basar, "Large intelligent surface-based index modulation: A new beyond MIMO paradigm for 6G," arXiv preprint arXiv:1904.06704, 2019. Available: https://arxiv.org/abs/1904.06704.

[4] C. Huang, A. Zappone, G. C. Alexandropoulos, M. Debbah, and C. Yuen, "Reconfigurable intelligent surfaces for energy efficiency in wireless communication," IEEE Trans. Wireless Commun., vol. 18, no. 8, pp. 4157-4170, Aug. 2019.
[5] Q. Wu and R. Zhang, "Intelligent reflecting surface enhanced wireless network: Joint active and passive beamforming design," in IEEE Global Commun. Conf. (GLOBECOM), Dec. 2018, pp. 1-6.

[6] _ "Beamforming optimization for intelligent reflecting surface with discrete phase shifts," in 2019 IEEE Int. Conf. Acoustics, Speech and Signal Processing (ICASSP), May 2019, pp. 7830-7833.

[7] _ " "Intelligent reflecting surface enhanced wireless network via joint active and passive beamforming," IEEE Trans. Wireless Commun., vol. 18 , no. 11, pp. 5394-5409, Nov. 2019.

[8] S. Hu, K. Chitti, F. Rusek, and O. Edfors, "User assignment with distributed large intelligent surface (LIS) systems," in 2018 IEEE 29th Annual Int. Symp. Personal, Indoor and Mobile Radio Commun. (PIMRC), Sep. 2018, pp. 1-6.

[9] M. Cui, G. Zhang, and R. Zhang, "Secure wireless communication via intelligent reflecting surface," IEEE Wireless Commun. Letters, pp. 1-1, 2019.

[10] E. Basar, "Transmission through large intelligent surfaces: A new frontier in wireless communications," in 2019 European Conf. Networks and Commun. (EuCNC), Jun. 2019, pp. 112-117.

[11] Y. Han, W. Tang, S. Jin, C. Wen, and X. Ma, "Large intelligent surfaceassisted wireless communication exploiting statistical CSI," IEEE Trans. Veh. Technol., vol. 68, no. 8, pp. 8238-8242, Aug. 2019.

[12] M. Di Renzo, M. Debbah, D.-T. Phan-Huy, A. Zappone, M.-S. Alouini, C. Yuen, V. Sciancalepore, G. C. Alexandropoulos, J. Hoydis, H. Gacanin et al., "Smart radio environments empowered by reconfigurable AI meta-surfaces: An idea whose time has come," EURASIP Journal on Wireless Commun. and Networking, vol. 2019, no. 1, p. 129, 2019.

[13] Q. Wu and R. Zhang, "Towards smart and reconfigurable environment: Intelligent reflecting surface aided wireless network," IEEE Commun. Mag., vol. 58, no. 1, pp. 106-112, Jan. 2020.

[14] A. Taha, M. Alrabeiah, and A. Alkhateeb, "Enabling large intelligent surfaces with compressive sensing and deep learning," arXiv preprint arXiv:1904.10136, 2019. Available: https://arxiv.org/abs/1904.10136.

[15] F. Benkhelifa, A. Tall, Z. Rezki, and M. Alouini, "On the low SNR capacity of MIMO fading channels with imperfect channel state information," IEEE Trans. Commun., vol. 62, no. 6, pp. 1921-1930, Jun. 2014.

[16] W. Liu, Y. Liang, Y. Li, and B. Vucetic, "Backscatter multiplicative multiple-access systems: Fundamental limits and practical design," IEEE Trans. Wireless Commun., vol. 17, no. 9, pp. 5713-5728, Sep. 2018.

[17] M. K. Simon, Probability distributions involving Gaussian Random Variables: a handbook for engineers and scientists. Springer, 2006.

[18] I. S. Gradshteyn and I. M. Ryzhik, Table of Integrals, Series and Products, 7th ed. Academic Press Inc, 2007.

[19] J. G. Wendel, "The non-absolute convergence of Gil-Pelaez' inversion integral," Ann. Math. Statist., vol. 32, no. 1, pp. 338-339, Mar. 1961.

[20] W. Zhao, G. Wang, S. Atapattu, C. Tellambura, and H. Guan, "Outage analysis of ambient backscatter communication systems," IEEE Commun. Lett., vol. 22, no. 8, pp. 1736-1739, Aug. 2018. 